\title{
Study of afterpulsing of MPPC with waveform analysis
}

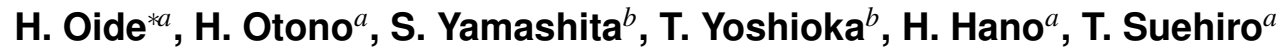 \\ ${ }^{a}$ Department of Physics, University of Tokyo \\ ${ }^{b}$ International Center for Elementary Particle Physics (ICEPP), University of Tokyo \\ 7-3-1 Hongo Bunkyo Tokyo Japan, 113-0033 \\ E-mail: oidedicepp.s.u-tokyo.ac.jp otonodicepp.s.u-tokyo.ac.jp \\ satorulicepp.s.u-tokyo.ac.jp tyosiokalicepp.s.u-tokyo.ac.jp \\ hanodicepp.s.u-tokyo.ac.jp suehirodicepp.s.u-tokyo.ac.jp
}

Study of afterpulsing events gives us an information of recovery time of Multi-Pixel Photon Counter (MPPC), which can be obtained by measuring the amplitude and the time interval between an afterpulse and its original pulse. It is important to measure the recovery time for investigating the internal mechanism of MPPC and to design detectors with this device. We have developed a waveform-analysis method to discriminate the pulses. As a result, recovery time and its bias dependency were obtained for three different types of MPPC.

International workshop on new photon-detectors PD07

June 27-29 2007

Kobe University, Kobe, Japan

\footnotetext{
${ }^{*}$ Speaker.
} 


\section{Introduction}

MPPC (Multi-Pixel Photon Counter) is a Geiger-mode avalanche photodiode (G-APD) made by Hamamatsu Photonics (HPK). The main attribute of G-APDs is that it is operated above the breakdown voltage, and its response to photon detection is constantly saturated. Thus the dynamic range of photon detection is assured by arranging same G-APDs in a line for one chip. The pulseheight of the signal is quasi-digitized, therefore we can "count" the number of photons detected by G-APDs.

G-APDs have a lot of advantages compared to conventional PMTs such as magnetic field immunity, low bias voltage, and their cost, thus they are expected to be alternate devices to PMTs. However, the noises are recognized as ones of the several issues to use G-APDs as a photon detector. Thus, characterization of noises (optical crosstalk, afterpulsing) has been studied actively for the last few years. First, we started studying the time characteristic of afterpulsing of MPPC by using waveform analysis, but we noticed that it is possible to probe the recovery time of MPPC using the afterpulsing. In this paper, we will present the recovery time and its bias dependency for three different types of MPPC.

\section{Afterpulsing and Recovery Time}

Once a pulse occurred by photon absorption or by internal thermal-electron excitation (thermal noise) in a pixel of MPPC, there is some possibility that another pulses are triggered incident to the original pulse.

Two different mechanisms are considered as the cause of these pulses[1]. First one is called optical crosstalk. When an original pulse is produced, the avalanching electrons at the pixel emit photons. Some of them could be absorbed by other pixels and they trigger another avalanches. This phenomenon is called optical crosstalk. The first characteristic of optical crosstalk is that the amplitude of crosstalked pulses is as same as normal avalanche, i.e. the pulse-height of crosstalked pulse is just one photoelectron $(1$ p.e.) response. The second characteristic is that the time difference between original and crosstalked pulses are quite short, and the original and crosstalked pulses happen "at the same time" in the range of our measurement precision. When we observe the waveform of crosstalked events using oscilloscope, we cannot distinguish original and crosstalked pulses, so the pulse-height of crosstalked events is 2 p.e., that is, as twice as single pulse.

Second one is called afterpulsing. The mechanism of this phenomenon is not so much clearer than optical crosstalk, generally it is considered that afterpulses are caused by avalanching electrons which are trapped at lattice defects in the semiconductor[1-5]. Once one of avalanching electrons is trapped, it is re-emitted after a certain time. This re-emitted electron causes another avalanche again, and it is recognized as an afterpulse. Afterpulsing should be distinguished from ordinary thermal noises in principle. By measuring the succeeding pulse rate, it is clearly recognized as an excess from the thermal noise rate[2].

Theoretically, trapping of avalanching electrons is caused in the same pixel that the original pulse was made just before. On the other hand, as MPPC is operated at Geiger-mode, once an pulse is caused, the bias voltage between anode and cathode goes down to the breakdown voltage $V_{0}$, and returns to operating voltage $V_{o p}$ in a short time. This duration is so-called recovery time. 


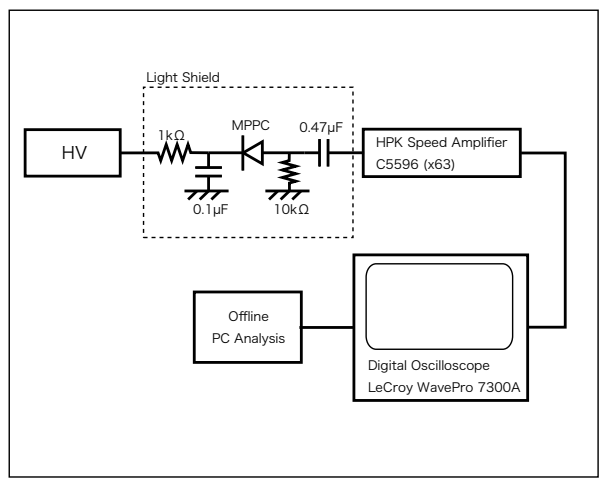

Figure 1: Measurement setup

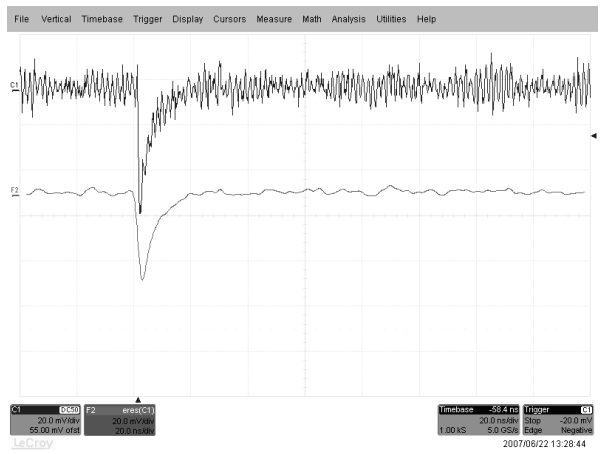

Figure 2: Raw waveform measured by oscilloscope (above) and filtered waveform using ERES mode (below) of 1600 px MPPC. Sampling rate is set to 200 [ps/sample] and ERES sampling number is set to 24. As for the ERES filter, see text.

If an afterpulse is caused during this recovery time, as the bias voltage is lower than $V_{o p}$, a pulse whose pulse-height is less than 1p.e. ought to be observed. Thus, by tracing the interval time and the amplitude of afterpulse, recovery curve can be obtained. Our purpose of this study is to probe the recovery process of MPPC by extracting afterpulsing events and to measure the recovery time. To measure the amplitude of afterpulsing event, waveform analysis is used since afterpulse close to its original pulse (typically less than 10 [ns]) is piled up on its original pulse.

Here we mention several issues. In case that a thermal noise is caused at the same pixel that the original pulse has been caused, we don't have any criterion to distinguish the thermal noise from afterpulsing. It is similar to the case that the thermal noises come after the recovery process has already ended. Statistical characteristic has a potential to solve this problem. As thermal noise rate is independent on time, it could be possible to extrapolate thermal noise distribution to the duration after the recovery process ended by measuring the thermal noise rate in the recovery process. The sensitivity of our analysis is mentioned in detail in Section 4.

\section{Measurement Setup}

The measurement was done for three different types of MPPC produced by HPK: $1600 \mathrm{px}, 400$ px, and 100 px. No light source was used and all triggered pulses are noises from the MPPC itself. The temperature is set around $300[\mathrm{~K}]$ (room temperature). We refer to the bias voltage later. The digital oscilloscope LeCroy WavePro 7300A was used for filtering and data storage. Measurement setup is shown in Figure 1.

In order to remove electrical noises and to extract waveform of pulses, appropriate sampling rate and filter were chosen. As it takes much time to filter noises from raw waveforms by off-line analysis, Enhanced RESolution (ERES) filter, which is internal function of the oscilloscope, was used. ERES filter looks like simple moving average filter, but the sample values are weighted, and the advantage of the ERES mode compared to moving average is that it produces a Gaussian frequency response [6]. Figrue 2 shows a sample of raw pulse signals and its filtered waveform using ERES. Generally speaking, by choosing higher sampling rate it is possible to get better time 


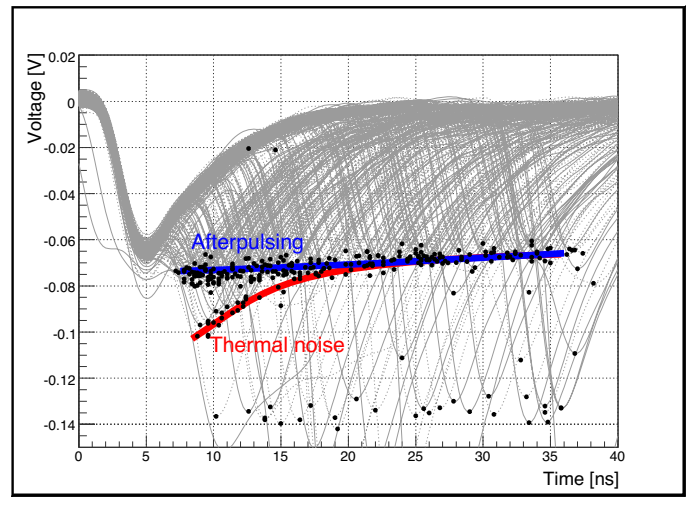

Figure 3: Superposition of events with the incidental pulses of 400 px MPPC (arranging the time origin to the peak of the original pulses)

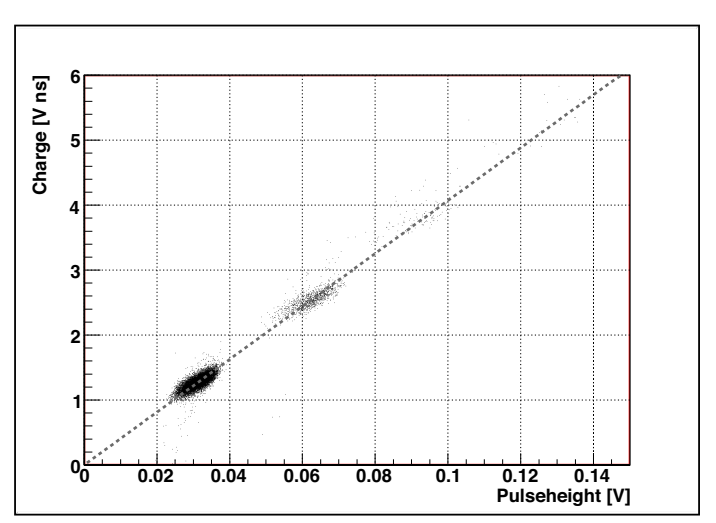

Figure 4: The correlation between charge and pulse-height of original pulse of each events

resolution for pulse discrimination, but it is necessary to use stronger smoothing filter for noise reduction, which reduces the time resolution. For $1600 \mathrm{px}$ and $400 \mathrm{px}$ MPPC, sampling number of ERES filter was set to 24 and for $100 \mathrm{px}$ it was set to 51 . Then, we select an appropriate sampling rate that eliminate almost all electrical noises. For 1600 px and 400 px MPPC we set the sampling rate to 200 [ps/sample] and for $100 \mathrm{px}$ we set to 400 [ps/sample].

\section{Waveform Analysis}

\subsection{Event Classification}

The method to extract the events that have the incidental pulses (afterpulses or thermal noises) is as follows. Firstly to eliminate crosstalked events, set two thresholds at 0.5 p.e. and 1.5 p.e., and extract events whose first pulse-height are between these two thresholds. Secondly, count the number of peaks in the event. If the number of peak is two or more, the event is classified as "event with incidental pulses". If the number of peak is one, there is two possibilities: one is the case that there is no incidental pulses (single event), and the other is that the incident pulses come after first pulse decayed below the threshold line. In this case another event search is executed. If there are other pulses, the event is classified as "event with incidental pulses", and otherwise it is classified as "single event". In this study we extracted only "single events" and "events with one incidental pulse". "Single events" are used for charge estimation of incidental pulses, and "events with one incidental pulse" are what we analyze. We neglected other events, and as a result the ratio of thermal noise to afterpulse was not measured accurately. Since our purpose is to measure recovery time, we didn't consider this issue in this study.

In this way only events with one incidental pulse are extracted. Figure 3 shows the superposition of these events with arranging the time origin to the peak of the original pulses. The dots indicate the peak points of incidental pulses. Remarkably, there are two distributions (blue and red lines) that converge to same line after about 20 [ns]. Apparently this suggests the existence of two different mechanisms for incidental pulses. Measuring the vertical difference between these two distributions and the decay curve of single events, we see the pulse-height of incidental pulses 

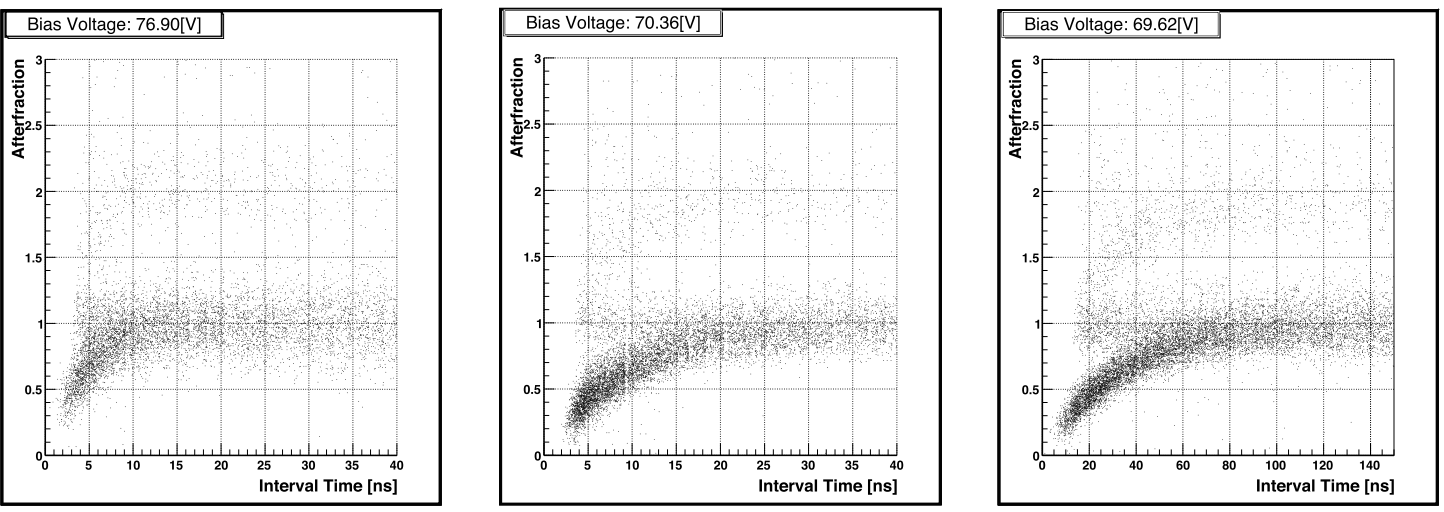

Figure 5: Recovery curve of different type MPPCs. (Left: 1600 px, Center: 400 px, Right: 100 px)

distributed to upper branch (blue line) increasing as time. This is the characteristic of afterpulsing that we mentioned in section 2 On the other hand, the pulse-height of those distributed to lower branch (red line) is constant to time. This is the characteristic of thermal noise.

\subsection{Recovery Curve Calculation}

To estimate the charge of incidental pulses, firstly it is required to calculate the mean charge of single events. As it is considered that the charge is proportional to the pulse-height within a few photoelectrons, it is possible to estimate first charge from its the pulse-height, that is immediately given from the waveform. Figure 4 shows the correlation between charge and pulse-height of first pulse of each events. By fitting the distribution to proportional line, the coefficient $\mathrm{c}$ is obtained as described later in detail.

After these procedures, we cope with recovery. Firstly define $f(t)$ as a particular waveform which is classified as "events with one incidental pulse". Determine the segment of the event $\left[t_{0}, t_{1}\right]$ so that whole pulse charges are included in this segment. Define total charge $Q_{\text {all }}$ as the integral of waveform: $Q_{a l l} \equiv \sum_{t_{i}=t_{0}}^{t_{1}} f\left(t_{i}\right)$. Define $t_{\text {orig }}$ as the peak position (time) of original pulse and $t_{i n c}$ as the peak position of incidental pulse. Measure the pulse-height of the original charge $f\left(t_{\text {orig }}\right)$, and using the proportionality between pulse-height and charge, i.e. $Q_{\text {orig }}=c f\left(t_{\text {orig }}\right)$, where $c$ represents the coefficient of proportionality, and subtracting the original charge from total charge we can estimate the charge of incidental pulse $Q_{i n c}=Q_{\text {all }}-Q_{\text {orig }}$. Then the quantity $\rho \equiv Q_{\text {inc }} / Q_{\text {org }}$ is plotted as a function of the interval time $T \equiv t_{i n c}-t_{\text {orig. }}$. We named the quantity $\rho$ afterfraction. If the incidental pulse is afterpulse, $\rho$ represents the time evolution of recovery and it approaches to unity asymptotically as recovery process ends. If the incidental pulse is thermal noise, the expectation value of $\rho$ is constantly unity. If the incidental pulse is just a electrical fluctuation, it is expected that $\rho$ distributes around 0 . This can be removed by imposing appropriate cutoff condition to $\rho$.

\section{Results}

We measured the recovery curve for $1600 \mathrm{px}$ MPPC in three different bias voltages, that is 2.8 [V], $3.3[\mathrm{~V}], 4.2[\mathrm{~V}]$ above the breakdown voltage $V_{0}=73.6[\mathrm{~V}]$. Slicing these scattering plot in interval time and calculating the most frequent value of $\rho$, and fitting these plot as a function 
$\bar{\rho}(T)=1-\exp (-T / \tau)$. The obtained parameter $\tau$ represents the recovery time. The result is for $1600 \mathrm{px}, \tau \simeq 4[\mathrm{~ns}]$ and we found it does not exhibit significant change as a function of the bias voltage.

We repeated the same approach for 400px and 100px as well. The results are summarized in Table 1.

We investigate the recovery time and the decay time of the single pulse, both of them are supposed to be related to the RC time constant of MPPC. As shown in Table 1, the recovery time and the pulse decay time are found to be consistent each other as expected.

Table 1: Recovery time and pulse decay time for three different types of MPPC

\begin{tabular}{lccc}
\hline Type & $1600 \mathrm{px}$ & $400 \mathrm{px}$ & $100 \mathrm{px}$ \\
\hline Bias voltage [V] & $V_{0}+3.3$ & $V_{0}+2.7$ & $V_{0}+0.87$ \\
Recovery time [ns] & $\sim 4$ & $\sim 9$ & $\sim 33$ \\
Pulse decay time [ns] & $\sim 5$ & $\sim 11$ & $\sim 35$ \\
\hline
\end{tabular}

\section{Conclusion}

We have developed a new method to measure the recovery time of the MPPC by analyzing a waveform of pulses caused by thermal noise. With this method the recovery time of the 3 types of MPPC has been measured. We found that the recovery time does not change by the bias voltage, and is strongly correlated with the RC constant of the MPPC. It is possible to improve the analysis method by using pulse-height of incidental pulses as their amplitude, which reduces the effect of the fluctuation of ground level, that will realize more precise recovery time estimation.

\section{References}

[1] D. Renker, Geiger-mode avalanche photodiodes, history, properties and problems, Nucl. Instrum. Methods Phys. Res., Sect. A, 5672006 48-56

[2] A.C. Giudice, M. Ghioni, S. Cova, A process and deep level evaluation tool: afterpulsing in avalanche junctions, European Solid-State Device Research, 2003. ESSDERC '03. 33rd Conference on 16-18 Sept. 2003 347-350

[3] S. Cova, G. Ripamonti, Trapping Phenomena in Avalanche Photodiodes on Nanosecond Scale, IEEE Elec. Dev. Lett., 12 (12) 1991

[4] Y. Kang, H.X. Lu, Y.-H. Lo, D.S. Bethune, W.P. Risk, Dark count probability and quantum efficiency of avalanche photodiodes for single-photon detection, Appl, Phys. Lett., 83 (14) 2003

[5] R.H. Haitz, Mechanisms contributing to the noise pulse rate of avalanche diodes, J. Appl. Phys., 36 19653123

[6] LeCroy LAB 767 - ERES vs. Boxcar Averaging http://www.lecroy.com/tm/library/registerPDF.asp?wp=625 